Aviation Associated Barotraumatic Pneumolabyrinth: A rare cause of Sudden Onset Hearing Loss
WHS
Guy's and St Thomas'
NHS Foundation Trust
S. Khosla, M. Whittaker, C. Skilbeck
Guy's and St. Thomas' NHS Foundation Trust

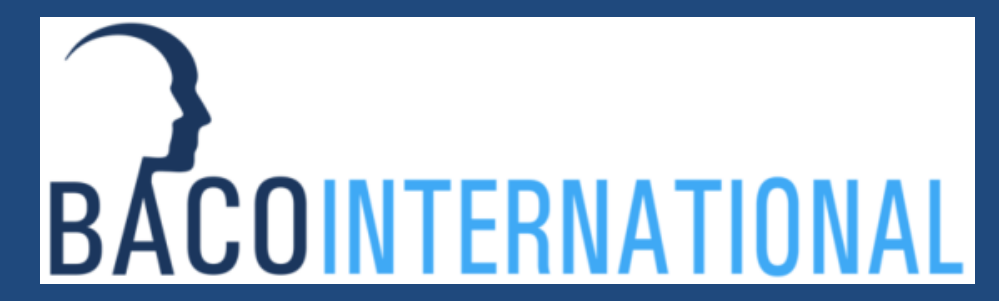

\title{
INTRODUCTION
}

Bilateral sudden onset hearing loss $(\mathrm{SOHL})$ is life changing. We present a novel case of SOHL due to barotraumatic pneumolabyrinth after air travel.

\section{PRESENTATION OF CASE}

Patient X presented with sudden onset hearing loss in the left ear, unsteady gait and horizontal nystagmus following an international flight.

He had a history of pansinusitis and had an ongoing upper respiratory tract infection prior to flying.

Two months previously a similar event had caused severe sensorineural hearing loss(SNHL) in the contralateral ear.

This resulted not only in sensory isolation and inability to work but also disabling vestibular hypofunction.
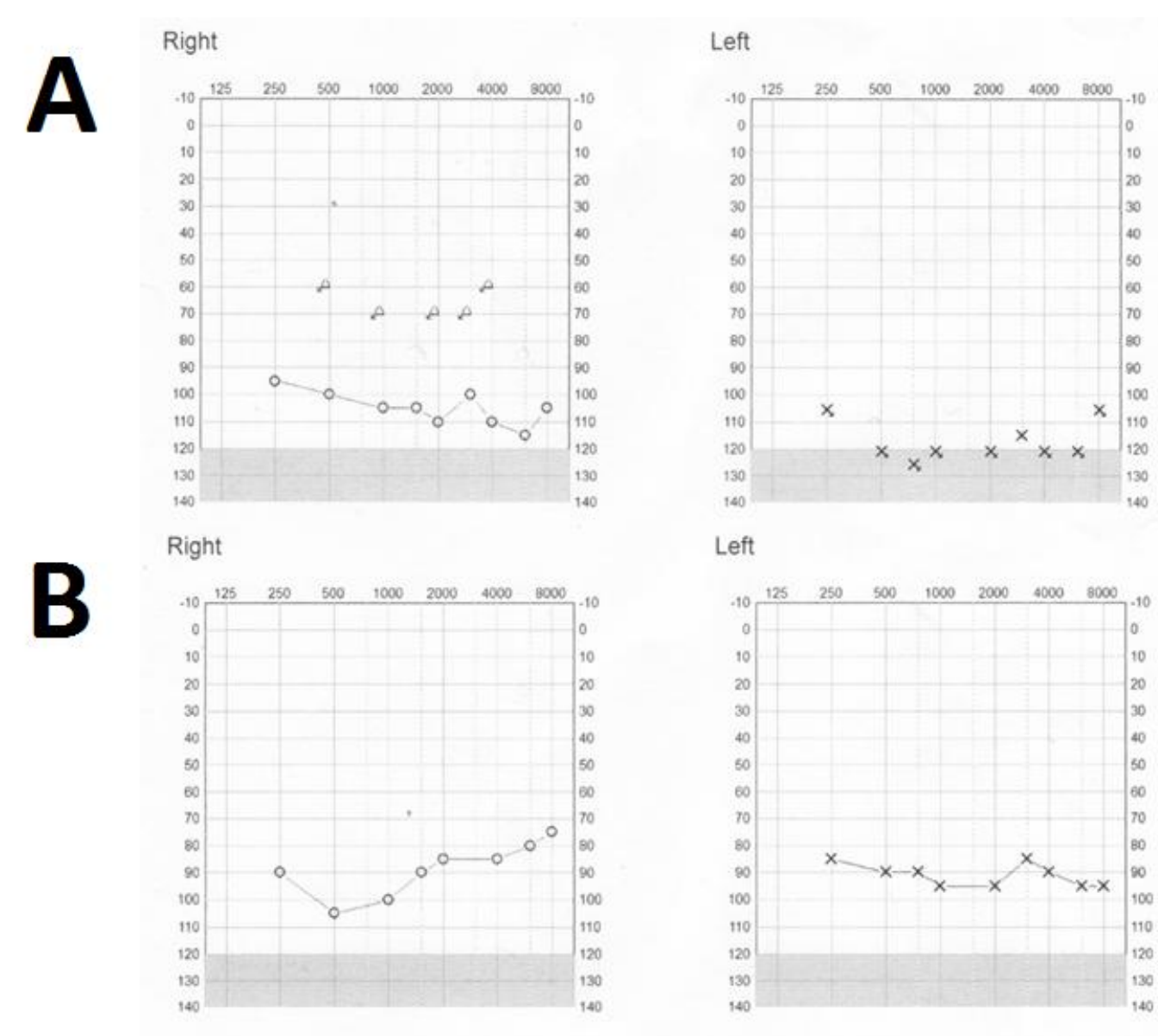

Figure 1: Pure tone audiometry (A) At presentation of symptoms and (B) Two months after exploratory tympanotomy.

\section{MANAGEMENT}

Haematological and biochemical investigations were normal.

Pure tone audiometry showed profound SNHL (Fig 1A).

Computed Tomography revealed gas replacing the endolymph of the cochlear and semicircular canals indicative of an oval or round window fistula (Fig. 2A). MRI showed no lesions involving the VIIth or VIIIth cranial nerves
A trial of high dose oral steroids and intratympanic dexamethasone resulted in no improvement.

Therefore the patient underwent exploratory tympanotomy which identified a round window defect (Fig. 3)

$>$ This was plugged with perichondrium and methylprednisolone soaked gelfoam placed to support the round window

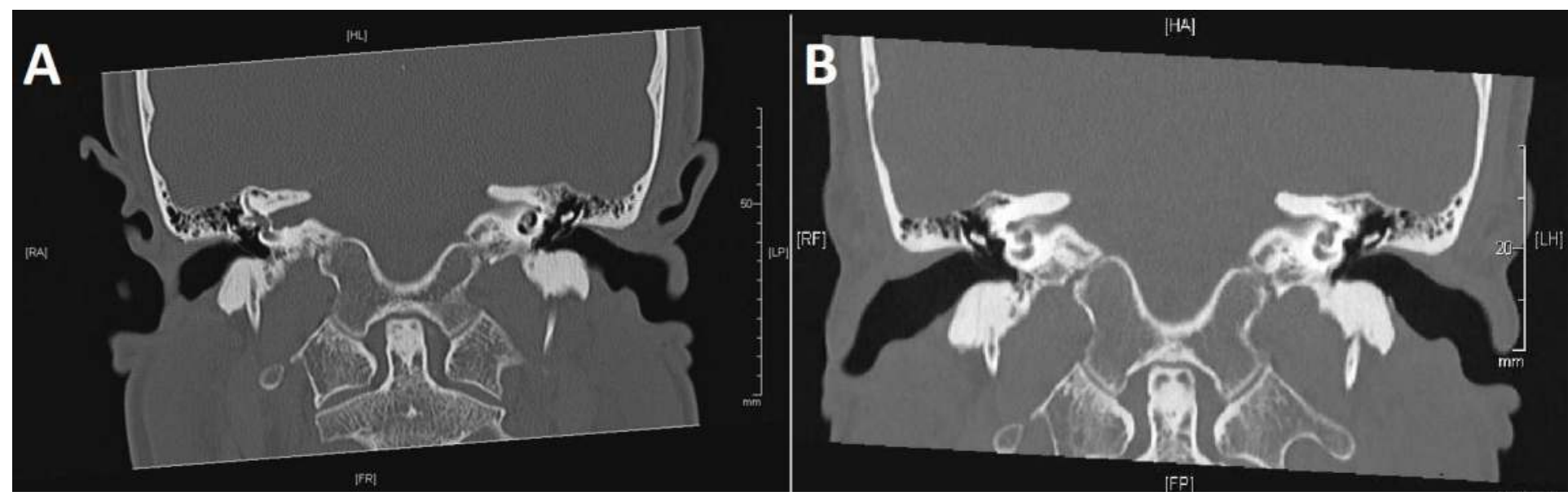

Figure 2: Coronal Computed Tomography of Mastoid showing evolution of pneumolabyrinth (A) At presentation of left sided symptoms (B) Two months after exploratory tympanotomy.

\section{PROGRESS}

Vestibular physiotherapy, speech therapy and psychological therapy has resulted in a significantly improved quality of life.

Repeat imaging revealed resolution of the pneumolabyrinth however repeat PTA showed minimal improvement(Fig. $1 \mathrm{~B}$ and $2 \mathrm{~B})$

The patient was therefore referred for consideration of cochlear implantation

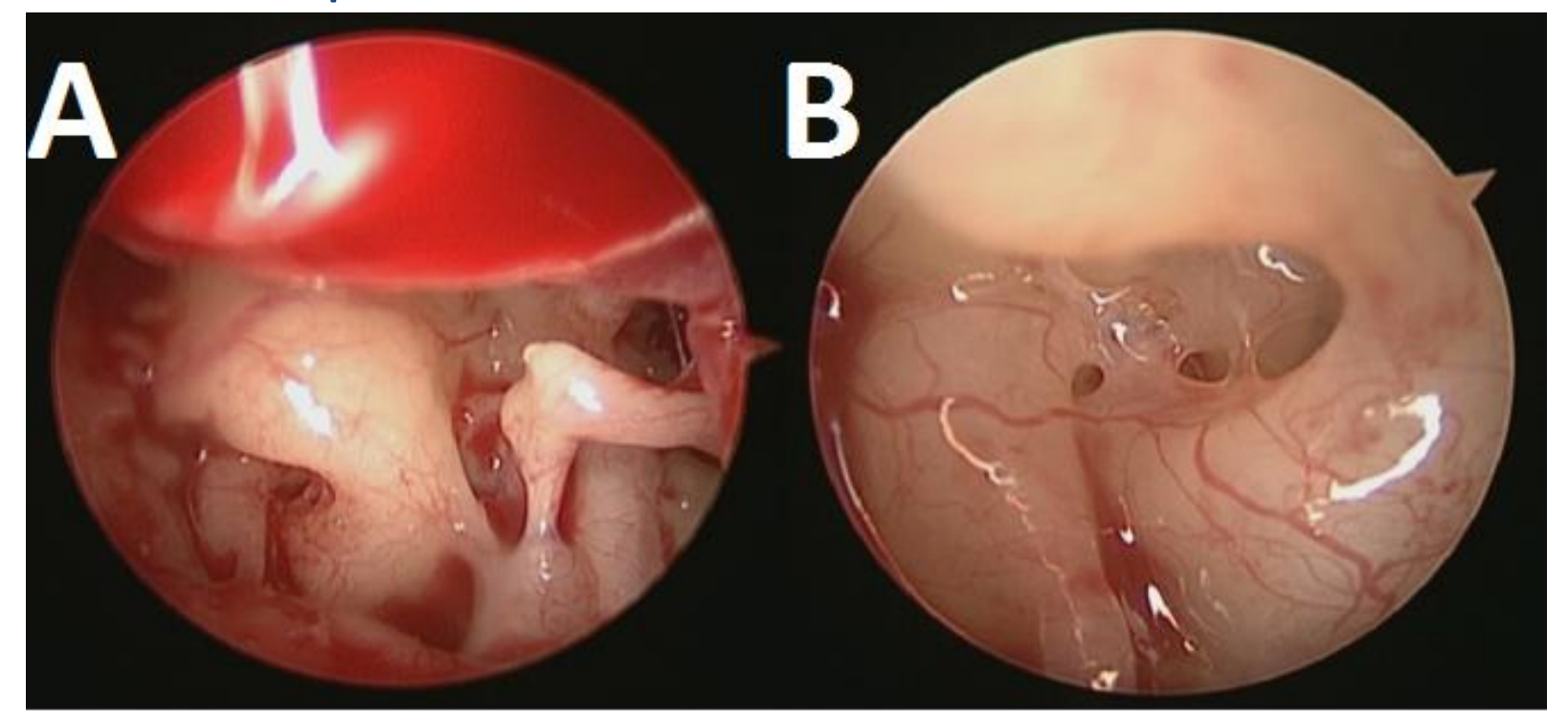

Figure 3: Endoscopic intraoperative photographs. (A) View of the posterior middle ear including the incudostapedial joint. (B) Defect in the round window niche with likely associated pseudomembrane.

\section{DISCUSSION}

Pneumolabyrinth secondary to aviation induced barotrauma is unreported.

Surgical intervention facilitated resolution of the pneumolabyrinth, however, long term effect on hearing is unclear.

The patient is now undergoing follow up after cochlear implantation 